Meta

Journal des traducteurs

Translators' Journal

\title{
La linguistique dans la formation des traducteurs arabes
}

\section{Jarjoura Hardane}

Volume 50, numéro 1, mars 2005

Enseignement de la traduction dans le monde

Teaching Translation Throughout the World

URI : https://id.erudit.org/iderudit/010664ar

DOI : https://doi.org/10.7202/010664ar

Aller au sommaire du numéro

Éditeur(s)

Les Presses de l'Université de Montréal

ISSN

0026-0452 (imprimé)

1492-1421 (numérique)

Découvrir la revue

Citer cet article

Hardane, J. (2005). La linguistique dans la formation des traducteurs arabes. Meta, 50(1), 137-144. https://doi.org/10.7202/010664ar

\section{Résumé de l'article}

L'itinéraire de l'enseignement de la traductologie à l'ÉTIB (École de Traducteurs et d'Interprètes de Beyrouth) permet de montrer l'utilité de la compétence théorique dans la formation à la traduction et le rôle que peuvent jouer les cours de linguistique, aux côtés des cours de traductologie, dans l'acquisition de cette compétence théorique. Cette acquisition est susceptible d'être assurée par des cours de traductologie et de linguistique conçus et donnés en arabe, à condition qu'ils utilisent des termes élaborés selon les paramètres scientifiques et consacrés par leur utilisation dans la production, même réduite, de textes linguistiques et terminologiques originairement écrits en arabe. 


\title{
La linguistique dans la formation des traducteurs arabes
}

\author{
JARJOURA HARDANE \\ Université Saint-Joseph, Beyrouth, Liban \\ jhardane@usj.edu.lb
}

\begin{abstract}
RÉSUMÉ
L'itinéraire de l'enseignement de la traductologie à l'ÉTIB (École de Traducteurs et d'Interprètes de Beyrouth) permet de montrer l'utilité de la compétence théorique dans la formation à la traduction et le rôle que peuvent jouer les cours de linguistique, aux côtés des cours de traductologie, dans l'acquisition de cette compétence théorique. Cette acquisition est susceptible d'être assurée par des cours de traductologie et de linguistique conçus et donnés en arabe, à condition qu'ils utilisent des termes élaborés selon les paramètres scientifiques et consacrés par leur utilisation dans la production, même réduite, de textes linguistiques et terminologiques originairement écrits en arabe.
\end{abstract}

\section{ABSTRACT}

Teaching translation studies at ÉTIB (École de Traducteurs et d'Interprètes de Beyrouth) shows the importance of the theoretic competence in the translator's training and the role that can be played through courses in linguistics and translation studies in the acquisition of this theoretic competence. This acquisition is best portrayed through courses in translation studies and linguistics developed and taught in Arabic, provided that they use the terminology elaborated according to scientific parameters and that this terminology is admitted by the users in the limited production of content-specific texts in linguistics and translation studies written in Arabic.

\section{MOTS-CLÉS/KEYWORDS}

arabe, théorie, enseignement de la traductologie, linguistique, interdisciplinarité

\section{Introduction}

Le problème de la place que devrait occuper la linguistique dans la formation des traducteurs et des interprètes ne date pas d'aujourd'hui. Il fut posé à la naissance même des programmes des premières institutions d'enseignement de la traduction et de l'interprétation comme disciplines académiques autonomes préparant à des métiers professionnellement identifiés et reconnus. Et il ne cesse jusqu'à nos jours de susciter chez les traductologues, les enseignants et les étudiants deux questions fondamentales: À quoi sert un cursus de linguistique dans une formation à la traduction ou à l'interprétation? Et quels en devraient être le contenu, la progression et la méthode? Nous essaierons d'y répondre à partir de l'historique des cours de linguistique à l'École de Traducteurs et d'Interprètes de Beyrouth qui assure la formation d'étudiants arabophones ayant bien entendu choisi l'arabe comme première langue de leur combinaison linguistique. L'objectif de notre réponse est double: montrer d'une part pourquoi des cours de linguistique dans les programmes de traduction et d'interprétation suivis par des étudiants arabophones posent des problèmes spécifiques; et dégager d'autre part les conditions que ces cours doivent remplir pour être utiles. 


\section{Linguistique et traduction: cohabitation généralement problématique}

L'introduction des cours de linguistique dans n'importe quel cursus de formation à la traduction et à l'interprétation pose principalement le double problème de leur utilité et de leur légitimité.

L'argument invalidant l'utilité de ces cours, et le plus souvent avancé par les étudiants eux-mêmes, paraît en effet logique et convaincant: de même que pour conduire une voiture, il n'est pas nécessaire de connaître le fonctionnement de son moteur, pour réussir une opération de traduction ou d'interprétation, il suffit de maîtriser l'utilisation des deux langues de départ et d'arrivée, sans pour autant décrire le fonctionnement de leurs systèmes phonétiques, morphologiques ou syntaxiques. Or, la linguistique se propose principalement de procéder à " une étude scientifique » de ces différents systèmes de la langue (Martinet, 1967: 6). Autant l'étudiant considère qu'il a nécessairement besoin de l'acquisition des systèmes «implicites» pour comprendre et s'exprimer clairement dans les deux langues de départ et d'arrivée, autant il voit dans l'acquisition, voire dans l'élaboration personnelle des systèmes «explicites» de l'une et de l'autre langue une pure perte de temps et d'énergie. Par conséquent, il accepte volontiers les cours de langue, que celle-ci soit la troisième, la deuxième et même la première dans la combinaison choisie (Hardane, 2000: 475), parce qu'ils en facilitent la compréhension et l'expression, et il refuse les cours de linguistique, parce qu'ils restent confinés à une pure description théorique n'apportant aucun secours dans l'opération même de traduction ou d'interprétation.

Cependant, l'argument classique en faveur des retombées positives que peut avoir la double démarche d'explicitation et de théorisation proposée par les cours de linguistique sur le terrain pratique de l'opération traduisante ne peut rester sans effet sur la conception des programmes des premières écoles de traducteurs et d'interprètes; d'abord parce que l'un des premiers objectifs de la fondation de ces écoles était d'assurer aux étudiants, non seulement un savoir-faire pragmatique et occasionnel que n'importe quel amateur pourrait acquérir par l'exercice, mais encore une formation théoriquement et méthodologiquement structurée qui tranche avec les approches artisanales, tâtonnantes et éparpillées; ensuite, parce que la linguistique, forte de son essor vertigineux d'une part et de sa crédibilité d'autre part, était la plus naturellement apte à assurer cette formation scientifiquement structurée dans l'un des domaines de communication qu'elle pouvait facilement revendiquer tels que celui de la traduction et de l'interprétation. C'est ainsi que des cours de linguistique, de volume, de contenu et de progression variable apparurent dans les programmes de la plupart des écoles à travers le monde.

Mais la légitimité de cette mission de théorisation et de structuration, assumée très tôt par la linguistique dans la formation à la traduction et à l'interprétation et contribuant considérablement à la naissance et au développement de ce que tout le monde appelle aujourd'hui la traductologie, fut dès le point de départ d'une remise en question (Mounin, 1963: 10-17). L'argument principal avancé à l'encontre de cette légitimité consiste à rappeler la nature même de l'opération traduisante: bien que se référant aux systèmes des deux langues en contact, elle se fixe comme objectif fondamental de reformuler dans la langue d'arrivée un acte de parole produit dans la langue de départ. Or, un acte de parole ne se réduit pas à ses éléments purement 
linguistiques, il renvoie à des dimensions culturelles, cognitives et contextuelles multiples (Lederer, 1994). Il ne saurait donc constituer un objet d'étude exclusivement réservé à la linguistique qui, selon Ferdinand de Saussure, aurait la principale tâche d'étudier la langue "qui est sociale dans son essence et indépendante de l'individu», et non la parole, «partie individuelle du langage» et dont l'étude reste «secondaire» (De Saussure, 1979: 37). Si la théorisation et l'explicitation s'avèrent indispensables à la traduction et à l'interprétation, elles doivent se faire en dehors de la linguistique et servir d'éléments constitutifs d'une traductologie reconnue comme discipline spécifique et autonome.

\section{La linguistique dans la formation des traducteurs arabes: problèmes spécifiques}

Dans le cas de la formation des traducteurs arabes, aux problèmes d'utilité et de légitimité s'ajoute le problème de la faisabilité. En effet, les cours de linguistique dans un cursus où l'arabe est choisi comme première langue devraient en principe être donnés en arabe, d'autant plus que tout au long de l'itinéraire de formation il est prévu que les productions réflexives et théorisantes, aboutissant en cinquième année à la préparation et à la soutenance d'un mémoire et nécessitant un éclairage indispensable assuré par la linguistique, se fassent exclusivement en langue arabe. Or, rien n'est moins évident, même aujourd'hui, plus d'un demi-siècle après l'apparition de la linguistique moderne dans les universités arabes, qu'un cours de linguistique, générale ou appliquée, conçu et assuré en arabe.

Il est vrai que la tradition linguistique arabe, notamment dans les domaines de la grammaire, de la phonétique et de la lexicographie, est plus que millénaire. Elle peut même être considérée, avec la tradition poétique, comme l'un des traits caractéristiques du bagage culturel arabe. Mais un double constat s'impose, dès qu'il s'agit de faire le bilan de la rencontre de la linguistique moderne avec cette tradition. D'un côté l'accueil réservé par les chercheurs, les enseignants et les étudiants arabes à cette nouvelle approche se constituant en discipline autonome et se proposant d'étudier «scientifiquement» les divers phénomènes du langage humain fut plutôt enthousiaste. Dès les années 50 , des cours de linguistique figurèrent dans les départements de lettres anglaises, françaises ou arabes. Des traductions en arabe d'ouvrages de référence en français ou en anglais et des articles ou même des ouvrages rédigés en arabe furent publiés. Des étudiants choisirent de se spécialiser en linguistique. Des dizaines de thèses en linguistique générale ou appliquée furent préparées et soutenues dans les universités américaines, européennes ou arabes. Mais d'un autre côté tous ces efforts individuels et collectifs ne réussirent pas à enclencher un mouvement irréversible susceptible de durer et de se développer. Un demi-siècle plus tard, la linguistique est toujours vécue comme une discipline «importée » et "étrangère», se débattant dans un double problème terminologique et culturel.

Il serait faux de croire que les termes spécifiques de la linguistique renvoyant à de nouvelles notions eurent du mal à trouver des équivalents en arabe. Bénéficiant d'une longue tradition de la langue arabe dans l'assimilation des termes étrangers grâce aux mécanismes exceptionnels de dérivation, traducteurs et linguistes ne tardèrent pas à proposer les équivalents adéquats. En moins d'une ou de deux décennies, la terminologie linguistique arabe offrait aux chercheurs, enseignants et étudiants la 
possibilité de lire et d'écrire en arabe des articles et des ouvrages de linguistique. Mais dès le point de départ elle souffrait de deux handicaps qui finirent par la paralyser. Le premier est l'instabilité due à la prolifération de plusieurs équivalents pour un seul terme. C'est ainsi que le terme phonème, pour ne citer qu'un exemple, eut droit à plus de trois équivalents dont aucun ne réussit à s'imposer. Il serait facile d'imputer cette instabilité à l'absence d'organismes unificateurs et normalisateurs dans les pays arabes, qui serait responsable bien entendu de ce désordre que connaissent toutes les terminologies modernes spécialisées introduites dans ces pays à l'époque moderne par la traduction ou l'adaptation, ce qui d'ailleurs est vrai. Mais la véritable raison est à rechercher dans un deuxième handicap dont continue à souffrir la terminologie linguistique: c'est celui du manque d'usage des équivalents qu'elle propose. Le problème est donc moins technique que culturel. La faiblesse de l'impact de la linguistique moderne sur la langue arabe provient moins d'une simple difficulté lexicale inhérente à toute tentative de forger des néologismes que d'un manque d'utilisation fréquente de ces néologismes. Les nouveaux schèmes de pensée engendrent de nouvelles attitudes, de nouvelles notions et par conséquent de nouveaux termes sont encore loin d'être adoptés et de rentrer définitivement dans l'usage oral et écrit de l'arabe. En linguistique, comme dans d'autres domaines relevant des sciences exactes ou des sciences humaines, seule une productivité prenant en compte à la fois l'apport moderne novateur et les éléments toujours opérationnels de la tradition est susceptible d'aboutir à une arabisation cohérente foncièrement tournée vers l'avenir. Or, il est facile de constater même aujourd'hui l'absence presque totale d'une telle productivité (Hardane: 2002).

\section{Les cours de linguistique à l'École de Traducteurs et d'Interprètes de Beyrouth}

On comprend dès lors pourquoi l'École de Traducteurs et d'Interprètes de Beyrouth, s'alignant sur les écoles qui avaient introduit un cursus de linguistique dans leurs programmes, malgré les problèmes d'utilité et de légitimité, proposa dès sa fondation en 1980 des cours de linguistique assurés en langue française. C'est ainsi que furent proposés un cours de phonétique et de phonologie en première année, un cours de syntaxe en deuxième année et un cours de sémantique en troisième année. Il est vrai que les enseignants illustraient régulièrement leurs propos par des exemples empruntés aussi bien au français qu'à l'arabe et qu'ils pratiquaient continuellement, dans leurs explications et leurs commentaires, un va-et-vient entre les deux langues. Mais les nouvelles approches et les nouvelles notions étaient principalement introduites par leurs appellations françaises et, faute de productions à la fois crédibles et accessibles en arabe, les sources, les références et les recherches utilisées étaient presque toujours empruntées au français. Quoi qu'il en soit, le malaise ressenti vis-à-vis des cours de linguistique amena les responsables, dix ans après la fondation de l'École, à les suspendre.

Un bilan s'imposait, d'autant plus que la décision consistait à suspendre les cours et non à les supprimer: ce qui fut fait pendant une période d'interruption qui dura presque une demi-décennie. Aux constats d'inutilité, d'illégitimité et de nonfaisabilité de ces cours, s'ajoutait le constat de leur inadaptation aux besoins réels d'un étudiant en traduction ou en interprétation. En effet, les cours de phonétique, 
de syntaxe et de sémantique proposaient les mêmes contenus et les mêmes approches méthodologiques et pédagogiques généralement adoptés dans n’importe quel cursus de linguistique s'inscrivant dans une pure formation à la linguistique générale ou dans une formation aux lettres classiques ou modernes. Aucun effort n'avait été fait pour les adapter aux exigences d'une formation à la traduction et à l'interprétation. Dans la conception de cette première version des programmes, on pouvait à peine déceler l'intention d'assurer aux étudiants par l'intermédiaire de ces cours la capacité de mener une réflexion théorisante sur l'opération traduisante. Mais en réalité cet objectif n'était jamais entièrement atteint. L'absence de cette capacité réflexive et théorisante était d'ailleurs attestée dans les mémoires de traduction préparés et soutenus jusqu'à cette époque et qui souffraient principalement de trois carences: la simplicité des problématiques se réduisant le plus souvent à la litanie des "problèmes rencontrés» dans la traduction, la faiblesse de l'approche théorique se contentant généralement de «quelques remarques » à propos de ces "problèmes rencontrés » et la difficulté de rendre compte de tout cela dans un texte entièrement écrit en arabe qui ne «sente pas le traduit».

Ces trois carences provenaient de l'avis même des étudiants en fin de parcours de l'absence d'une compétence théorique d'une part et d'un outillage terminologique en arabe d'autre part, préalables à l'étude en arabe de toute problématique traductologique. Cependant, malgré l'interruption des cours de linguistique, trois activités revêtant un triple aspect théorique, problématique et terminologique étaient maintenues. Aux côtés d'un cours de préparation à la méthodologie du mémoire toujours programmé en dernière année d'études, des sessions intensives de terminologie étaient annuellement organisées, alors que des projets rassemblant enseignants et étudiants dans le cadre du nouveau Centre d'Études et de Recherches en Terminologie Arabe enrichissaient lentement et partiellement, mais selon les méthodes modernes les plus fiables, la terminologie des différents domaines de la traductologie arabe par les équivalents aux nouveaux termes qui ne cessaient d'affluer vers l'arabe des langues déjà bien rodées à cette nouvelle discipline. Or, ces différentes activités étaient à la fois fortement demandées et très bien accueillies par les étudiants. Il ne restait plus qu'à les consolider, à leur accorder le volume d'heures qu'elles méritaient et à les intégrer définitivement aux programmes. C'est ce qui fut fait dès le milieu des années 90 , avec la proposition de deux cours qui venaient s'ajouter au cours de «méthodologie du mémoire» : celui de «lieux de production» initiant les étudiants à la terminologie et celui de "pédagogie de la traduction» traitant les problèmes traductologiques et pédagogiques de la traduction.

C'est alors que réapparut le besoin de cours de linguistique générale, à la lumière d'un nouvel éclairage sur la problématique posée par les rapports entre la linguistique et la traduction. Il s'avérait évident d'une part qu'il n'y avait pas d'incompatibilité entre les deux objets d'études de la linguistique et de la traductologie. Avec l'évolution des approches de la linguistique, on était loin, en ce milieu des années 90, de la scission entre langue et parole, et de la préférence saussurienne de la langue, au détriment de la parole. La parole était devenue un terrain privilégié de la linguistique qui avait élargi son investigation aux dimensions discursives et pragmatiques. Il devenait donc légitime que l'opération traduisante soit incluse dans son champ d'études. Il s'avérait évident d'autre part que la traductologie était née essentiellement dans l'enceinte de la linguistique et qu'elle lui devait bon nombre de ses approches 
théoriques et méthodologiques en même temps qu'une part importante de ses notions et de ses termes. Par ailleurs, ces éléments communs ne nuisaient en rien à l'autonomie de la traductologie qui s'affirmait de plus en plus, comme une science ayant un objet d'étude, une méthodologie et une terminologie spécifiques.

Et c'est ainsi que l'École de Traducteurs et d'Interprètes de Beyrouth se voyait prête à fixer ses choix théoriques et méthodologiques et à apporter des amendements cohérents et logiques à ces programmes : ce qu'elle fit sans trop tarder. Considérant d'abord que la traductologie était devenue une discipline autonome, elle choisit de l'enseigner en tant que telle dans un cours intitulé «problèmes théoriques» et dans un «atelier de mémoire» débouchant sur la préparation d'un mémoire traitant principalement une problématique traductologique. Estimant ensuite qu'une formation minimale en linguistique et en terminologie était indispensable à ce cursus traductologique, elle réorganisa l'enseignement de la terminologie qui ne s'était jamais interrompu en deux cours: celui de «dictionnaires II » dont l'objectif était d'assurer une initiation à la terminologie et celui de "problèmes terminologiques » qui faisait le tour des problématiques terminologiques posées en traduction. Elle rétablit surtout l'enseignement de la linguistique en introduisant trois cours. L'objectif des deux premiers, intitulés «linguistique et traduction I» et «linguistique et traduction II », était double: faire acquérir aux étudiants les notions de base de la linguistique, et notamment celles reprises par la traductologie, et leur faire découvrir la place qu'occupe la linguistique dans l'itinéraire de la traductologie. Le second, intitulé «dictionnaires 1 », se proposait d'assurer aux étudiants les notions sémiologiques, sémantiques et lexicologiques susceptibles de les préparer d'une part à une meilleure évaluation, et à une meilleure utilisation du dictionnaire dans l'opération traduisante, et de leur fournir d'autre part les outils théoriques et méthodologiques nécessaires à une éventuelle participation aux différents chantiers lexicographiques, spécialement en langue arabe.

Il apparaissait ainsi que le rétablissement des cours de linguistique faisait logiquement suite à deux changements majeurs dans la conception et l'application des programmes. Le premier s'était opéré à travers l'éclaircissement des rapports entre la linguistique, l'opération traduisante et la traductologie. Le résultat en avait été double: d'un côté, les éléments communs entre les trois domaines justifiaient la présence de la linguistique dans l'itinéraire de formation, et de l'autre, l'adaptation d'éventuels cours de linguistique à la spécificité de la formation devenait un choix indispensable. Le second changement s'était produit grâce à une nouvelle attitude de l'École face au problème terminologique arabe: au lieu de baisser les bras devant les difficultés d'élaboration, de normalisation et d'utilisation des nouveaux termes, elle choisit d'intensifier et de développer les projets terminologiques déjà lancés qui obéissaient aux normes scientifiques et répondaient à des besoins spécifiques comme celui de l'adaptation en arabe de la «terminologie de la traduction» qui vient d'être publiée. Les nouveaux outillages terminologiques en langue arabe, fruit de ces différents projets, et utilisés de plus en plus systématiquement dans les mémoires préparés par les étudiants et les interventions ou articles écrits par les chercheurs et les enseignants ouvraient la voie à des cours ou à des productions en linguistique, en terminologie et en traductologie conçus et assurés à part entière en langue arabe. 


\section{Conclusion}

L'itinéraire des cours de linguistique à l'École de Traducteurs et d'Interprètes de Beyrouth permet à l'observateur de faire à partir des événements vécus en l'espace de deux décennies trois généralisations. La première, c'est que la compétence réflexive et théorisante devrait faire partie intégrante d'une formation à la traduction et à l'interprétation (Delisle 1999: 49-69). Dans l'opération traduisante elle-même, elle est susceptible de guider l'étudiant vers l'essentiel et de lui faire éviter les dérapages du tâtonnement et les insuffisances du travail artisanal. Et dans les démarches de description, de systématisation et d'évaluation, elle lui fournit les outils méthodologiques et terminologiques adéquats.

La deuxième conclusion, c'est que cette compétence devrait relever obligatoirement de la nouvelle discipline qu'il est convenu d'appeler «traductologie» et qui réussit à s'imposer par un objet d'étude, une méthodologie et une terminologie spécifiques lui assurant un statut d'autonomie largement reconnu.

La troisième conclusion, c'est que les cours de linguistique s'inscriraient naturellement dans l'acquisition de cette compétence fixée comme objectif ultime de la traductologie. Vu les éléments communs entre la traductologie et la linguistique et vu le rôle de la linguistique dans la naissance et le développement de la traductologie, des cours de linguistique, adaptés et appropriés, seraient d'un grand secours aux cours de traductologie. Quoi qu'il en soit, la traductologie n'est pas seulement perméable à la linguistique: à l'image de toutes les sciences humaines, voire de toutes les sciences, elle ne pourrait être qu'interdisciplinaire.

\section{NOTES}

1. L'École de Traducteurs et d'Interprètes de Beyrouth, fondée en 1980 par les professeurs Roland MEYNET, SJ, et René CHAMUSSY, SJ, offre aux étudiants une combinaison linguistique constituée, à l'image du paysage des langues au Liban, de l'arabe comme première langue, du français comme deuxième et de l'anglais comme troisième. En fonction des itinéraires personnels des étudiants, le français peut figurer comme A', et l'anglais comme B.

2. Trois termes au moins continuent de circuler dans les textes arabes comme équivalents de "phonème » : le premier (مينف) est une pure transcription arabisée de "phonème", le deuxième est

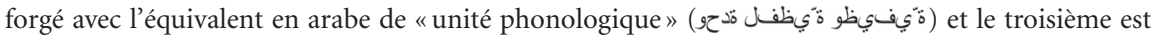

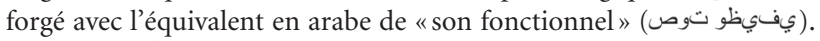

3. Le Centre d'Études et de Recherches en Terminologie Arabe (CERTA) a été fondé en 1996. Il a à son actif des recherches terminologiques sur les termes-clés dans le domaine de la linguistique, de la psychologie, de la maladie de la vache folle et de la maladie du charbon. Il a aussi activement contribué à la recherche qui a donné naissance à la version arabe de la Terminologie de la Traduction, ouvrage de Jean Delisle, Hannelore Lee-Jahnke et Monique C. Cormier publié sous les auspices de la CIUTI et de la FIT. Le CERTA travaille actuellement sur la terminologie de la terminologie. À noter que le CERTA encadre aussi les recherches terminologiques entreprises par les étudiants de l'ÉTIB.

4. C'est le fruit d'un travail en équipe qui visait à trouver les équivalents en arabe de la Terminologie de la traduction, élaborée sous la direction de Jean Delisle, Hannelore Lee-Jahnke et Monique C. Cormier dans le cadre de la FIT et de la CIUTI et publiée en français, en anglais, en allemand et en espagnol.

Ladaptation qui a obéi, face aux nouveaux termes, à deux critères essentiels, la proposition de nouveaux équivalents et l'adoption, lorsque cela s'avérait possible, des équivalents disponibles dans le bagage lexicographique arabe, donna lieu à une publication en arabe:

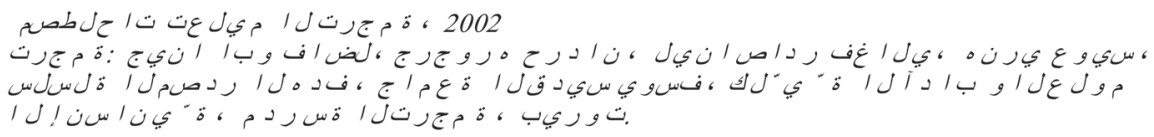




\section{RÉFÉRENCES}

Delisle, J. (1999) : «Utilité de la théorie en enseignement de la traduction», dans Traduction: Approches et Théories, collection «Sources - Cibles", École de Traducteurs et d'Interprètes, Faculté des Lettres et des Sciences Humaines, Beyrouth, Université Saint-Joseph.

Hardane, J. (2000): «La formation du traducteur arabe: cas de la mise à niveau linguistique», dans META 45-3, Montréal, Les Presses de l'Université de Montréal.

Hardane, J. (2002): «Impact et authenticité en traduction, le cas de la traduction linguistique du français vers l'arabe», dans Du pareil au même, l'auteur face à son traducteur, actes du colloque organisé par l'ÉTIB et l'Instituto Cervantes, collection «Sources-Cibles», École de Traducteurs et d'Interprètes, Faculté des Lettres et des Sciences Humaines, Beyrouth, Université Saint-Joseph.

Lederer, M. (1994): La traduction aujourd'hui, le modèle interprétatif, Paris, Hachette.

Martinet, A. (1967): Eléments de Linguistique générale, Paris, A. Colin.

Mounin, G. (1963): Les problèmes théoriques de la traduction, Paris, Gallimard.

SAussure, F. DE (1979): Cours de linguistique générale, Paris, Payot. 